\title{
Acute Correction of Varus Knee by Biplanar Medial Opening-Wedge High Tibial Osteotomy and Fixation with TomoFix Plate
}

\author{
Elsayed Shaheen, Mohamed Abdel-Aal Morsy \\ Orthopedic Surgery Department, Faculty of Medicine, Al-Azhar University, Cairo, Egypt \\ Email: ssshaheen2007@yahoo.com
}

How to cite this paper: Shaheen, E. and Morsy, M.A.-A. (2019) Acute Correction of Varus Knee by Biplanar Medial OpeningWedge High Tibial Osteotomy and Fixation with TomoFix Plate. Open Journal of Orthopedics, 9, 89-100.

https://doi.org/10.4236/ojo.2019.94009

Received: February 8, 2019

Accepted: April 1, 2019

Published: April 4, 2019

Copyright (อ 2019 by author(s) and Scientific Research Publishing Inc. This work is licensed under the Creative Commons Attribution International License (CC BY 4.0).

http://creativecommons.org/licenses/by/4.0/

\begin{abstract}
Background: Biplanar medial opening wedge high tibial osteotomy (MOWHTO) is a treatment method that allows adequate correction of symptomatic varus knee deformity. However, MOWHTO tends to decrease patellar height and increase the posterior tibial slope, which can affect the knee joint stability. Objective: The aim of this study was to describe the technique of biplanar MOWHTO and fixation by TomoFix plate, as well as to evaluate the accuracy of the planned correction and the postoperative tibial slope. Patients and Methods: This prospective cohort study was conducted on patients who presented with varus knee deformity and underwent biplanar MOWHTO and fixation by TomoFix plate during the period from March 2016 to March 2017. Assessment of patients included pre- and postoperative Knee and function scores, mechanical femorotibial angle (mFTA), posterior tibial slope angle (pTSA), range of motion (ROM) and radiological evaluation of the healing of the osteotomy site. Results: The recruited patients were 13 ( 8 men and 5 women), with average age 31.7 years old. The knee and functional scores improved from the preoperative mean of 45 and 41 to the postoperative mean of 85 and 72 points, respectively. The average knee flexion was 115 degrees, which at the final follow-up remained unchanged except for one case. The mean preoperative mFTA was $13.5^{\circ}$ varus and decreased postoperatively to a mean of $3^{\circ}$ valgus. The average postoperative follow-up period was 12 months $(10-18 \mathrm{M})$. Conclusion: The biplanar MOWHTO allows preservation of posterior tibial slope while correcting the varus knee adequately.
\end{abstract}

\section{Keywords}

Varus Knee, Biplanar, Medial Opening-Wedge, High Tibial Osteotomy, 


\section{Introduction}

High tibial valgus osteotomy (HTO) is becoming an increasingly popular method to correct varus malalignment in patients with or without medial-compartment osteoarthritis $(\mathrm{OA})$ of the knee. This procedure is considered to be joint preserving, as when performed precisely it can delay or eliminate the need for joint replacement [1] [2]. The goal of biplanar HTO is to decrease the load in the medial compartment of the knee by transferring the mechanical axis slightly lateral to the midline of the joint, thereby delaying the development and progression of OA [3] [4] [5]. HTO surgery includes the lateral closed-wedge high tibial valgus osteotomy (CWHTO) [6] and medial open-wedge high tibial valgus osteotomy (OWHTO) [7].

The CWHTO procedure used to be the most commonly used technique of HTO [8]. However, many drawbacks have been reported for CWHTO, including lateral-offset increases (due to horizontal osteotomy), decreased bone block distal to the lateral tibial plateau and shortening of the operated side after the surgery (with subsequent discrepancies in leg lengths) [9] [10]. The CWHTO procedure may also be complicated by delayed union at the osteotomy site due to loss of opposition between the area on the proximal and distal fragments; therefore, the alignment should be maintained for a long time until bone healing is achieved.

Recently, the MOWHTO procedure has gained more popularity [11]due to its advantages. Compared to CWHTO, the MOWHTO is easier, maintains more bone block, and it avoids injury to the peroneal nerve, proximal tibiofibular joint and the anterior leg compartment [12].

The AO principles recommend stable fixation of osteotomy to allow early and safe mobilization. The TomoFix knee osteotomy system is a plating system that allows stable fixation of osteotomies at the knee joint by supporting fixed angle stability between the screw and plate [13].

The purpose of this study was to describe the technique of biplanar MOWHTO and fixation by TomoFix plate, as well as to evaluate the accuracy of the planned correction and the postoperative tibial slope.

\section{Patients and Methods}

This prospective study was conducted in the Andalusia hospitals during the period from the $1^{\text {st }}$ of March 2016 to the $1^{\text {st }}$ of March 2017. Patients suffering from varus knee deformity were included in this study and were treated with biplanar MOWHTO and internal fixation by TomoFix plate. Informed consent was obtained from the patients or their parents prior to the procedure. Confidentiality of patients' data was maintained by assigning a code number to each patient 
known only to the researchers.

Inclusion criteria: All patients who presented with symptomatic varus knee deformity, with or without medial gonarthrosis (idiopathic or posttraumatic varus deformity of the proximal tibia) were included in the study.

Exclusion criteria: Patients were excluded from the study if they had inflammatory arthritis, previous osteotomy of the same knee, bi-compartmental (medial and lateral) OA, fixed flexion contracture $>25^{\circ}$, restricted range of motion/fixed deformities (knee flexion $<90$ degrees), ligamentous instability, lateral tibial subluxation of more than $1 \mathrm{~cm}$ or medial compartment tibial bone loss of more than 2 or $3 \mathrm{~mm}$.

\subsection{Preoperative Planning}

Patients' characteristics, including age, gender, body mass index (BMI) and side, were recorded. The measurement of mechanical femorotibial angles (mFTAs) were obtained using plain anteroposterior radiographs with full-leg length, weight bearing view. The radiographs were taken with the patients standing on both legs and the knee joints fully extended.

The amount of required correction (target correction angle) was determined by drawing a line from Fugisawa point to the center of femoral head and another line to the center of knee joint [14] The Fugisawa point is located at $62.5 \%$ of the tibial plateau from the medial tibial articular margin [15].

\subsection{Surgical Technique}

The same surgical procedure was applied in all cases and biplanar osteotomy was carried out according to the technique recommended by the AO principles [9]. (Figure 1) In all cases, an ascending and transverse biplanar medial open wedge osteotomies were performed with the use of insertion bone grafts. Fixation by locked plates was done to stabilize osteotomy using TomoFix ${ }^{\oplus}$ system (Synthes, Solothurn, Switzerland) (Figure 2).

\subsection{Technical Details of the Biplanar MOWHTO Procedure}

Patient's position and incision: The patient was placed in supine position on a radiolucent operating table. The anatomic landmarks (medial joint line, upper border of pes anserinus, course of the medial collateral ligament and tibial tuberosity) were marked on the skin. A longitudinal incision, 6 to $7 \mathrm{~cm}$ in length, was performed midway between the tibial tuberosity (TT) and the posteromedial border of the tibia. The incision started half an inch distal to the joint line and was extended to the tendons of pes anserinus. A Hohmann retractor was inserted between the superficial medial collateral ligament and the posterior cortex of the tibia (Figure 3). The distal end of the patellar tendon was clearly visualized to allow planning of the ascending and transverse cuts of the biplanar osteotomy [16].

Biplanar medial opening wedge osteotomy: The starting point of osteotomy was the upper border of the pes anserinus. Under the c-arm image, two parallel 


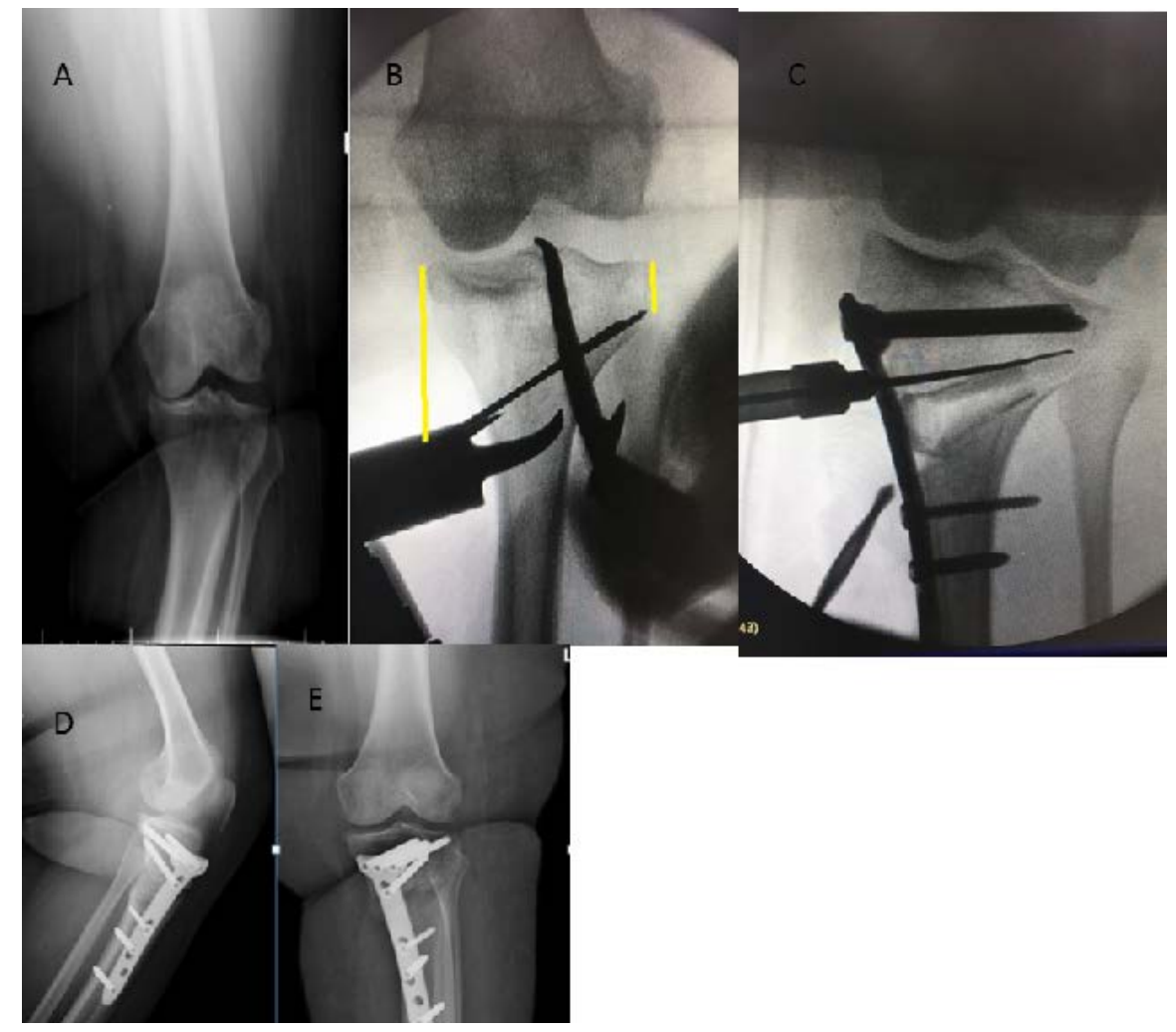

Figure 1. (A) AP standing view of knee with severe varus deformity; (B) Oblique k wire directed to head of the fibular without penetration to lateral cortex, arrow line $1 \mathrm{~cm}$ on lateral cortex from joint line; (C) Left knee, medial view Locking Tomfix HTO plate. Osteotomy gap filled with tricortical synthetic allograft; (D) Postoperative radiographic AP and lateral with correction of varus deformity Note that Measurement of posterior tibial slope has been made.

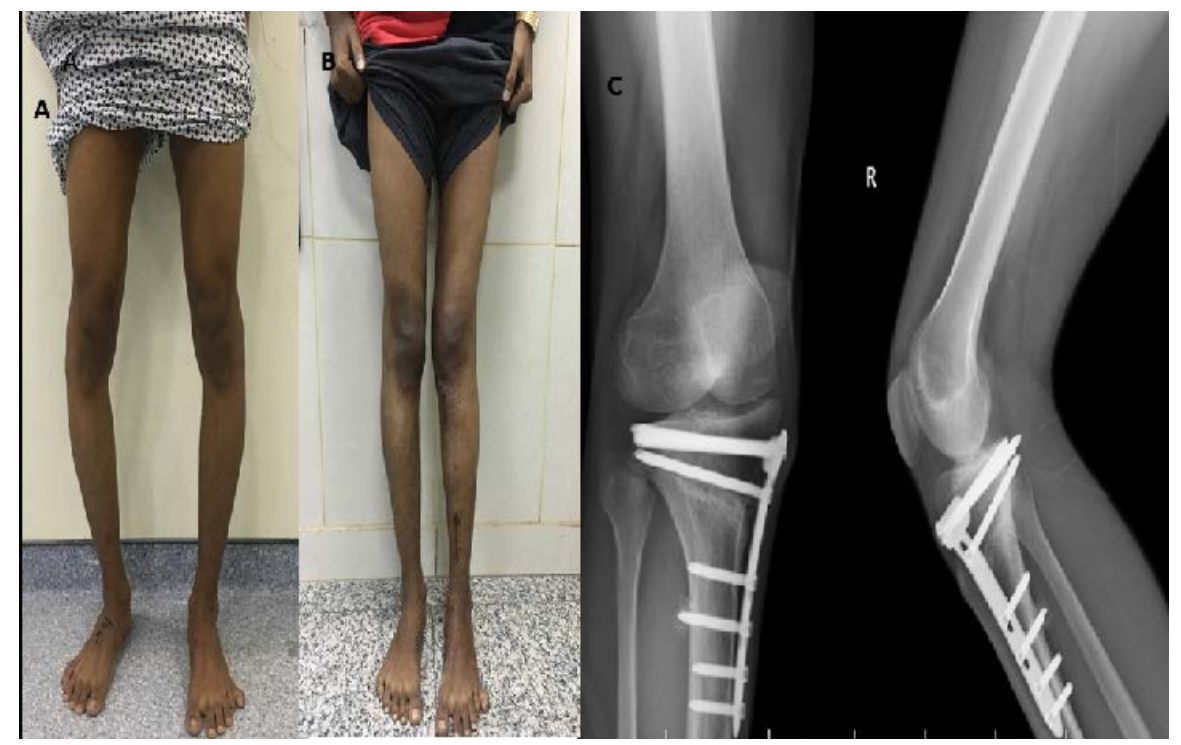

Figure 2. (A) (B) Photo view showing pre and postoperative biplanar osteotomy fixed by Tomofix plate both knee, right knee more than $1 \mathrm{y}$; (C) Postoperative X-ray right knee AP and LAT with overcorrection about $3^{\circ}$ with bone healing at osteotomy side and rigid plate. 


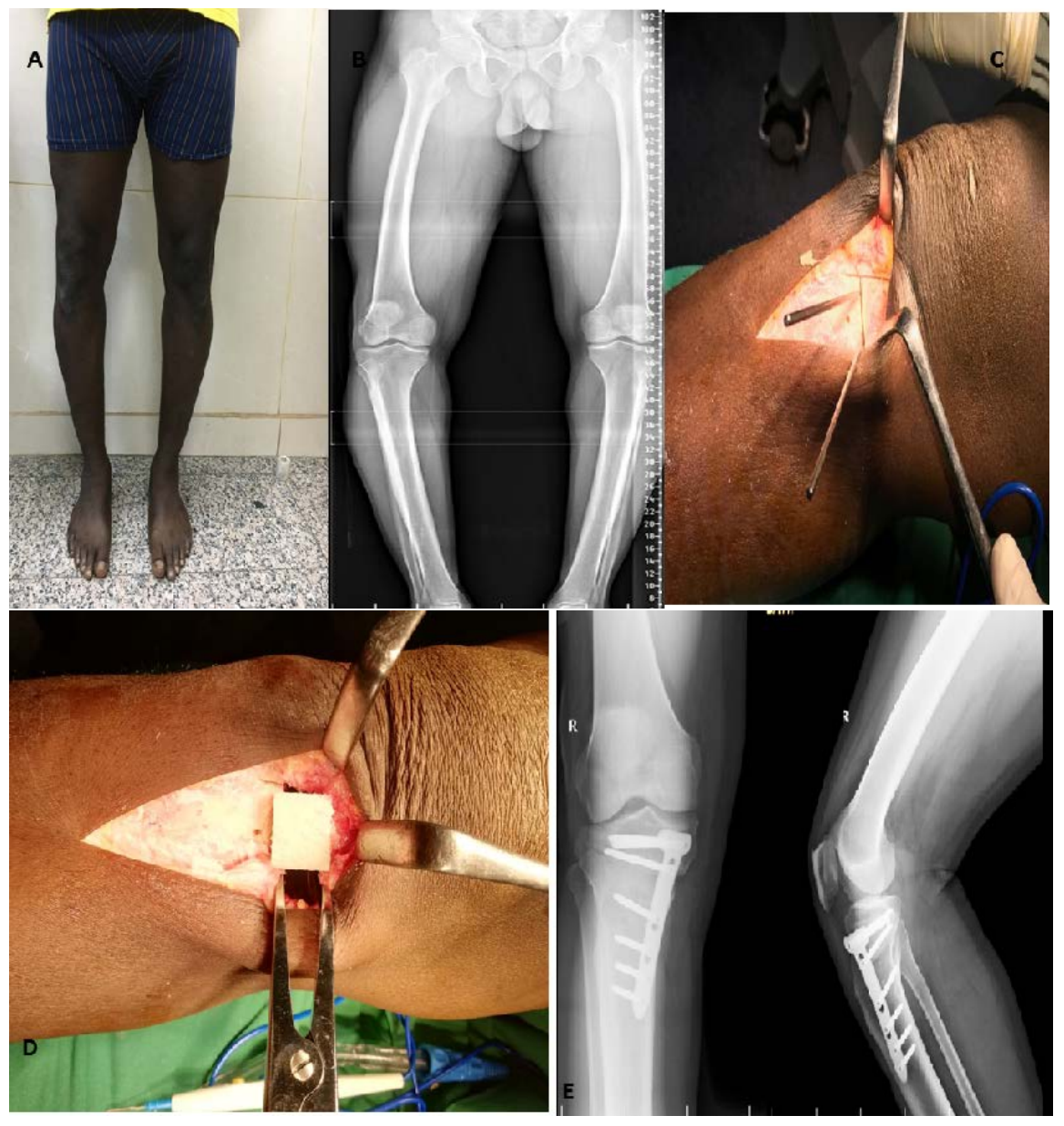

Figure 3. (A) Photo standing position showing Rt more varus knee; (B) Full-weight-bearing long-leg standing (Scanograms) anteroposterior (AP) radiographs showing severe varus knee on right sid; (C) Intraoperative photosho win: Right knee, medial view. A 2.4-mm K. guidewire is placed in the medial cortex of the tibia, aimed toward the tip of the fibular head in a proximal and posterolateral direction, both wires must run in parallel and aim towards the hinge point; (D) Osteotomy gap filled with tricortical iliac bone crest allograft (E) Postoperative X ray AP and LAT knee with corrected osteotomy and Tomofix plate, with slight increase of posterior tibial slope.

Kirschner wires were inserted obliquely directed from medial to the lateral hinge. The lateral hinged point was the upper end of fibular head. Care was taken to avoid penetration of the lateral cortex to preserve the hinged point [17]. The ascending osteotomy cut was made parallel to the anterior cortex of the tibial shaft about one inch behind the tibial tuberosity. The transverse cut was made parallel to tibial plateau to ensure good bony contact in the area of the ascending cut after opening the osteotomy $110^{\circ}$ to the transverse osteotomy [17] (Figure 3).

After achieving biplanar the osteotomies, the gap was spread gradually using chisels and a specially calibrated spreading device. When the measurement of the gap was acceptable and equal to the preoperative planned angle, the laminar spreader was inserted to save the realignment position until the cable line as- 
sessed the mechanical axis by connecting the hip center to the ankle center, passing through the knee center laterally [15] (Figure 3).

Filling of the osteotomy gap was achieved using allograft or autograft tricortical strut mixed with autologous bone marrow or bone chips. Under fluoroscopy, the prepared plate was inserted medially and the shaft portion was aligned with the tibial diaphysis, in order to prevent the occurrence of anterior or posterior cortical overhang. Eight locked holes were used in the TomoFix device (four proximal and four distal), with three of them being unicortical. Conventional plate was inserted firstly to contour the plate. In addition, another lag screw was inserted through the first distal hole below the osteotomy after proximal fixation in order to functionally compress the lateral hinged cortex of the osteotomy and to eliminate any potential instability in this area [17] (Figure 3).

\subsection{Post-Operative Care and Follow-Up Protocol}

Ice bag and intermittent venous compression were applied to relieve the swelling. Continuous passive motion (CPM) and muscle-strengthening exercises were started on the second day after surgery. Before six weeks, partial weight bearing with walker was allowed based on the amount of pain. After six weeks, full weight-bearing was allowed.

The final evaluations of the patients were performed by measuring the American Knee Society (AKS) knee and function scores, as well as assessing the mFTAs and changes in pTSA. The pTSA was measured between the medial tibial plateau and the posterior tibial cortex [17].

\subsection{Statistical Analysis}

Statistical analysis was carried out using SPSS for Windows (version 17.0; SPSS, Chicago, IL). Categorical data (gender, site, complications, graft used) were summarized as number and frequency. Numerical data were all normally distributed (as assessed by Shapiro Wilk test); they were summarized as mean \pm standard deviation $(\mathrm{M} \pm \mathrm{SD})$. Comparison between pre- and postoperative measurements was done using paired $\mathrm{T}$ test. Statistigal significance was considered at $\mathrm{p}$ value $<0.05$.

\section{Results}

Thirteen patients underwent biplanar MOWHTO during the study period, 8 men and 5 women. Their age ranged between 17 and 45 years old, with average age of $31.7 \pm 9.5$ years old. Their BMI ranged between 20.5 and 35, with average of $29 \pm 3.7 \mathrm{Kg} / \mathrm{m}^{2}$. Varus deformity affected the right knee in 6 patients $(46.2 \%)$, left knee in 4 patients (30.8\%) and both knees in 3 patients (23.1\%) (Table 1).

The knee score improved from the preoperative mean of 45 (range $32-60$ ) to postoperative mean of 85 points (range 49 - 92). The mean preoperative functional score was 41 (range $32-58$ ) and mean postoperative functional score was 72 points (range 52 - 93). The mean preoperative TFA was $13.5^{\circ}$ varus (range 7 - 
Table 1. Patients' characteristics. Age and BMI are summarized as mean \pm SD (range). Gender and side are expressed as number (\%).

\begin{tabular}{ccccc}
\hline Patient & Age & Gender & BMI & Side \\
\hline 1 & 34 & M & 35 & Bilat \\
2 & 19 & M & 34 & RT \\
3 & 30 & F & 28 & RT \\
4 & 25 & F & 26.5 & RT \\
5 & 18 & F & 26.5 & Bilat \\
6 & 45 & M & 27 & Bilat \\
7 & 39 & M & 28.5 & LT \\
8 & 42 & F & 30 & RT \\
9 & 17 & M & 29.5 & LT \\
10 & 38 & M & 31.5 & RT \\
11 & 40 & M & 20.5 & LT \\
12 & 29 & F & 31 & LT \\
13 & 36 & M & 29 & RT \\
Summary & $(17.0-45.0)$ & Female 5(38.5\%) & $(20.5-35.0)$ & Rt 6 (46.2\%) \\
statistics & $31.7 \pm 9.5$ & Male 8 (61.5\%) & $29.0 \pm 3.7$ & Bilat 3 $23.1 \%)$ \\
\hline
\end{tabular}

M: male; F: female; BMI: body mass index; Bilat: bilateral; Rt: right; Lt: left.

20) and decreased significantly after the surgery to a mean of $3.2^{\circ}$ valgus (range $-2-8 ; \mathrm{p}<0.001$ ). The preoperative average knee flexion was 121 (ranged from 100 to 140 ), which at the final follow-up remained unchanged except for one case that was complicated by limited full extension about $5^{\circ}$. Overcorrection was observed in one patient only. The mean pTSA changed significantly from $4^{\circ}$ preoperatively, to $6.5^{\circ}$ postoperatively $(\mathrm{p}=0.015)$. The mean time of bone union was at 4.7 months, with a range between 3 and 6 months after osteotomy. Correction was maintained for all patients postoperatively, except for one patient who presented by delayed union at 5 months and improved by knee brace and follow up until bone healing. Skin irritation occurred in one case only. The mean time to full weight-bearing was 5.6 weeks (range 4 - 8 weeks). Superficial wound infection occurred in one case and was treated successfully with appropriate antibiotics (Table 2).

\section{Discussion}

The current study demonstrates that acceptable correction of the mFTA was achieved by biplanar MOWHTO and fixation by TomoFix plate. Furthermore, the knee function improved significantly (improved knee and function scores) at the final follow-up. Previous studies have reported similar results.

The mean corrected angle of mFTA was 3.3 in our study, with a range between -2 to 8 degrees. The reported corrected angle of mFTA ranged from 6 to 
Table 2. Comparison of mean TFA and pTSA pre- and post-operatively, ROM, FW bearing, time of union, bone graft, and complications.

\begin{tabular}{|c|c|c|c|c|c|c|c|c|c|}
\hline \multirow{2}{*}{ Patient } & \multicolumn{2}{|c|}{ TFA } & \multicolumn{2}{|c|}{ pTSA } & \multirow{2}{*}{ ROM } & \multirow{2}{*}{ FW bearing } & \multirow{2}{*}{$\begin{array}{l}\text { Time of } \\
\text { union }\end{array}$} & \multirow{2}{*}{ Bone graft } & \multirow{2}{*}{ Complications } \\
\hline & Pre & Post & Pre & Post & & & & & \\
\hline 1 & 7 & 1 & 5 & 7.2 & 100 & 8 & 4 & Auto & \\
\hline 2 & 12.5 & 3 & 2.5 & 8 & 110 & 4 & 5 & Synth & skin irritation \\
\hline 3 & 13 & 4 & 3 & 12 & 105 & 6 & 6 & Synth & \\
\hline 4 & 15.5 & 6 & 5.4 & 9.7 & 120 & 5 & 3 & Auto & \\
\hline 5 & 15 & 5 & 3.5 & 4 & 130 & 6 & 4 & Synth & \\
\hline 6 & 20 & 7 & 4 & 5.5 & 120 & 7 & 5 & Synth & \\
\hline 7 & 13.5 & 0 & 6 & 6.5 & 135 & 4 & 4 & Auto & limited extension \\
\hline 8 & 10 & -2 & 4 & 3 & 120 & 4 & 5 & Synth & \\
\hline 9 & 10 & 1 & 5 & 3.5 & 115 & 5.5 & 6 & Synth & \\
\hline 10 & 9.5 & 6 & 4.5 & 4 & 125 & 6.5 & 4.5 & Synth & superficial infection \\
\hline 11 & 16 & 8 & 3 & 10 & 140 & 6.5 & 3.5 & Synth & overcorrection \\
\hline 12 & 17 & 2 & 2.8 & 9 & 125 & 5.5 & 6 & Synth & \\
\hline 13 & 16 & 1 & 5.7 & 6.7 & 130 & 4.5 & 5 & Synth & \\
\hline Summary & $\begin{array}{c}13.5 \pm 3.6 \\
(7.0-20.0)\end{array}$ & $\begin{array}{c}3.2 \pm 3.0 \\
(-2.0-8.0)\end{array}$ & $\begin{array}{c}4.2 \pm 1.2 \\
(2.5-6.0)\end{array}$ & $\begin{array}{c}6.9 \pm 2.8 \\
(3.0-12.0)\end{array}$ & $\begin{array}{c}121 \pm 12 \\
(100-140)\end{array}$ & $\begin{array}{c}5.6 \pm 1.3 \\
(4.0-8.0)\end{array}$ & $\begin{array}{c}4.7 \pm 1.0 \\
(3.0-6.0)\end{array}$ & $\begin{array}{l}\text { Auto 3 } \\
(23.1 \%)\end{array}$ & \\
\hline statistics & \multicolumn{2}{|c|}{$\mathrm{P}<0.001^{*} \#$} & \multicolumn{2}{|c|}{$\mathrm{p}=0.015^{\star} \#$} & $\mathrm{p}=0.039^{\star}$ & $\mathrm{p}=0.039^{\star}$ & $\mathrm{p}=0.042^{\star}$ & $\begin{array}{c}\text { Synth } 10 \\
(76.9 \%)\end{array}$ & \\
\hline
\end{tabular}

TFA: tibio-femoral angle; pTSA: posterior tibial slope angle; Pre: preoperative; Post: postoperative; ROM: range of motion; FW: full weight; Synth: synthetic; \#: paired $\mathrm{T}$ test; ${ }^{*}$ significant at $\mathrm{p}<0.05$.

14 degrees with good clinical outcomes. Overcorrection occurred in one patient only in the present study. Over and undercorrection were reported by previous studies, with contradictory clinical results. Hernigou et al. [18] reported that an overcorrection of more than $6^{\circ}$ was associated with progressive articular degeneration; an undercorrection was associated with poor patient satisfaction and increased the symptoms of the medial compartment OA. On the contrary, overcorrection of the mFTA was recommended by Ivarsson et al. [19]. Rudan and Simurda [20] found that overcorrection (with the mFTA above $15^{\circ}$ ) seemed to achieve excellent clinical results, but the patient was not satisfied cosmetically; undercorrection was associated with more failure of the procedure.

The average knee flexion in our patients remained unchanged till the final follow-up period, except for one case that had limited full extension that may be attributed to increased tibial slope in this case by about 10 degrees.

In the current study, bone graft was used in all patients, either tricortical synthetic (10 cases) or autologous ( 3 cases) grafts. Gaasbeek et al. [21] have observed that the TCP filler that was used with MOWHTO was absorbed, with the new bone being completely remodeled and incorporated into the native bone. In patients who are at increased risk of non-union, such as smokers and obese pa- 
tients, autologous graft from iliac bone are recommended to be used [21]. However, Staubli and Jacob [22] and Zaki and Rae [23] reported the achievement of good bone healing without bone graft or substitute in a TomoFix plate group.

The common drawbacks of MOWHTO were evaluated in this study and their incidence was low, including skin irritation, limited extension, superficial infection and overcorrection, which occurred in one case each. Changes in tibial slope are the most common drawbacks reported by previous studies. Tibial slope is crucial for maintaining the knee joint stability and biomechanics [24]. Generally, tibial slope tends to increase after OWHTO and to decrease after CWHTO [25]. The increase in tibial slope is due to the anatomical characteristics of the proximal tibia. It was found that an opening wedge with equal heights of the anterior and posterior gaps could increase the sagittal tibial slope [26] [27]. Increased pTSA may disturb the corrected coronal plane [28] and could result in more tension on cruciate ligament and destabilization of the knee joint [29] [30]. Tibial slope changes might also progress to early OA. In order to reduce the increase in tibial slope, the anterior opening gap is recommended to be $1 / 2$ to $2 / 3$ of the posterior gap [26] [27].

The undercorrection in the frontal plane in cases of severe varus deformity was shown by Asada et al. [28] to increase pTSA. We found that pTSA was maintained postoperatively. Other previous studies have also reported an increase of PTSA after OWHTO that ranged from 2 to 4 degrees [28] [31] [32] [33]. TomoFix plate fixation allowed for maintenance of pTSA in the sagittal plane. Proximal screws were fixed first, followed by a single screw in the distal plate. Thereafter, the pTSA was adjusted guided by fluoroscopy, with the knee joint in the extended position. Lastly, a screw was inserted in the remaining distal hole to complete the fixation.

Another reported drawback of MOWHTO is the decrease in patellar length which was suggested by Goutallier et al. [16]. This change results from destabilization and lateralization of the tibial tuberosity. Moreover, Amzallag et al. [34] showed that patellar height decreased more than $20 \%$ in one third of patients after OWHTO. Therefore, standard OWHTO is contraindicated in patients with patella baja or infera [34]. The patellar height was not affected in the present study.

There was no screw loosening or implant failure in this study as we used the TomoFix locking system. The use of TomoFix plate provided many advantages as it enabled rigid fixation, early range of motion, weight-bearing [28] and maintenance of pTSA [35] [36]. However, there are some disadvantage of TomoFix system that includes extensive skin irritation, prominent implant and soft tissue incision. Skin irritation was observed in one case in this study and was attributed to prominent implant and subcutaneous inflammation.

This study was subject to some limitations. The number of cases was small and follow-up period was short. Future studies should be conducted on larger sample size and with patient follow-up for longer duration. 


\section{Conclusion}

Biplanar MOWHTO accompanied with stable fixation using a TomoFix plate produced good clinical outcomes with low incidence of complications, without affecting patellar height or the posterior tibial slope. In order to minimize the rate of complications, surgeons must perform accurate planning before the surgery, follow the surgical technique meticulously and provide ample fixation using rigid stable implants.

\section{Conflicts of Interest}

The authors declare no conflicts of interest regarding the publication of this paper.

\section{References}

[1] Hofmann, A.A. and Kane, K.R. (1994) Total Knee Arthroplasty after High Tibial Osteotomy. Orthopedics, 17, 887-890.

[2] Keene, J.S. and Dyreby, J.J. (1983) High Tibial Osteotomy in the Treatment of Osteoarthritis of the Knee. The Role of Preoperative Arthroscopy. The Journal of Bone and Joint Surgery, 65, 36-42. https://doi.org/10.2106/00004623-198365010-00006

[3] Maquet, P. (1985) The Treatment of Choice in Osteoarthritis of the Knee. Clinical Orthopaedics and Related Research, 192, 108-112.

https://doi.org/10.1097/00003086-198501000-00013

[4] Akamatsu, Y., Koshino, T., Saito, T. and Wada, J. (1997) Changes in Osteosclerosis of the Osteoarthritic Knee after High Tibial Osteotomy. Clinical Orthopaedics and Related Research, 334, 207-214.

[5] Coventry, M.B. (1973) Osteotomy about the Knee for Degenerative and Rheumatoid Arthritis: Indications, Operative Technique, and Results. The Journal of Bone and Joint Surgery, 55, 23-48. https://doi.org/10.2106/00004623-197355010-00002

[6] Pape, D., Kohn, D., van Giffen, N., Hoffmann, A., Seil, R. and Lorbach, O. (2013) Differences in Fixation Stability between Spacer Plate and Plate Fixator Following High Tibial Osteotomy. Knee Surgery, Sports Traumatology, Arthroscopy, 21, 82-89. https://doi.org/10.1007/s00167-011-1693-8

[7] Brinkman, J.M., et al. (2008) Osteotomies around the Knee: Patient Selection, Stability of Fixation and Bone Healing in High Tibial Osteotomies. The Journal of Bone and Joint Surgery, 90, 1548-1557. https://doi.org/10.1302/0301-620X.90B12.21198

[8] Insall, J.N., Joseph, D. and Msika, C. (1984) High Tibial Osteotomy for Varus Gonarthrosis. A Long-Term Follow-Up Study. The Journal of Bone and Joint Surgery, 66, 1040-1048. https://doi.org/10.2106/00004623-198466070-00010

[9] Lobenhoffer, P. and Agneskirchner, J.D. (2003) Improvements in Surgical Technique of Valgus High Tibial Osteotomy. Knee Surgery, Sports Traumatology, Arthroscopy, 11, 132-138. https://doi.org/10.1007/s00167-002-0334-7

[10] van den Bekerom, M.P., et al. (2008) Early Complications After High Tibial Osteotomy-A Comparison of Two Techniques. The Journal of Knee Surgery, 21, 68-74. https://doi.org/10.1055/s-0030-1247797

[11] Elmalı, N., et al. (2013) Monoplanar versus Biplanar Medial Open-Wedge Proximal Tibial Osteotomy for Varus Gonarthrosis: A Comparison of Clinical and Radiolog- 
ical Outcomes. Knee Surgery, Sports Traumatology, Arthroscopy, 21, 2689-2695. https://doi.org/10.1007/s00167-012-2040-4

[12] Chae, D.J., et al. (2011) Early Complications of Medial Opening Wedge High Tibial Osteotomy Using Autologous Tricortical Iliac Bone Graft And T-Plate Fixation. The Knee, 18, 278-284. https://doi.org/10.1016/j.knee.2010.05.009

[13] DePuy Synthes Trauma (2017) TomoFix ${ }^{\text {Tm }}$ Medial High Tibial Plate (MHT): Surgical Technique. Synthes GmbH, Switzerland, 1-76.

[14] Rossi, R., Bonasia, D.E. and Amendola, A. (2011) The Role of High Tibial Osteotomy in the Varus Knee. Journal of the American Academy of Orthopaedic Surgeons, 19, 590-599. https://doi.org/10.5435/00124635-201110000-00003

[15] Fujisawa, Y., Masuhara, K. and Shiomi, S. (1979) The Effect of High Tibial Osteotomy on Osteoarthritis of the Knee. An Arthroscopic Study of 54 Knee Joints. The Orthopedic Clinics of North America, 10, 585-608.

[16] Goutallier, D., Delepine, G. and Debeyre, J. (1979) The Patello-Femoral Joint in Osteoarthritis of the Knee with Genu Varum (Author's Transl). Revue de Chirurgie Orthopedique et Reparatrice de 1 Appareil Moteur, 65, 25-31.

[17] Noyes, F.R., Barber, S.D. and Simon, R. (1993) High Tibial Osteotomy and Ligament Reconstruction in Varus Angulated, Anterior Cruciate Ligament-Deficient Knees: A Two-To Seven-Year Follow-Up Study. The American Journal of Sports Medicine, 21, 2-12. https://doi.org/10.1177/036354659302100102

[18] Hernigou, P., et al. (1987) Proximal Tibial Osteotomy for Osteoarthritis with Varus Deformity. A Ten to Thirteen-Year Follow-Up Study. The Journal of Bone and Joint Surgery, 69, 332-354. https://doi.org/10.2106/00004623-198769030-00005

[19] Ivarsson, I., Myrnerts, R. and Gillquist, J. (1990) High Tibial Osteotomy for Medial Osteoarthritis of the Knee. A 5 to 7 and 11 Year Follow-Up. The Journal of Bone and Joint Surgery, 72, 238-244. https://doi.org/10.1302/0301-620X.72B2.2312563

[20] Rudan, J.F. and Simurda, M.A. (1990) High Tibial Osteotomy. A Prospective Clinical and Roentgenographic Review. Clinical Orthopaedics and Related Research, 255, 251-256. https://doi.org/10.1097/00003086-199006000-00033

[21] Gaasbeek, R.D., et al. (2005) Mechanism of Bone Incorporation of $\beta$-TCP Bone Substitute in Open Wedge Tibial Osteotomy in Patients. Biomaterials, 26, 6713-6719. https://doi.org/10.1016/j.biomaterials.2005.04.056

[22] Staubli, A.E. and Jacob, H.A. (2010) Evolution of Open-Wedge High-Tibial Osteotomy: Experience with a Special Angular Stable Device for Internal Fixation without Interposition Material. International Orthopaedics, 34, 167-172. https://doi.org/10.1007/s00264-009-0902-2

[23] Zaki, S. and Rae, P. (2009) High Tibial Valgus Osteotomy Using the Tomofix Plate-Medium-Term Results in Young Patients. Acta Orthopaedica Belgica, 75, 360-367.

[24] Agneskirchner, J.D., et al. (2004) Effect of High Tibial Flexion Osteotomy on Cartilage Pressure and Joint Kinematics: A Biomechanical Study in Human Cadaveric Knees. Winner of the AGA-DonJoy Award 2004. Archives of Orthopaedic and Trauma Surgery, 124, 575-584. https://doi.org/10.1007/s00402-004-0728-8

[25] El-Azab, H., Halawa, A., Anetzberger, H., Imhoff, A.B. and Hinterwimmer, S. (2008) The Effect of Closed-And Open-Wedge High Tibial Osteotomy on Tibial Slope: A Retrospective Radiological Review of 120 Cases. The Journal of Bone and Joint Surgery, 90, 1193-1197. https://doi.org/10.1302/0301-620X.90B9.20688

[26] Noyes, F.R., Goebel, S.X. and West, J. (2005) Opening Wedge Tibial Osteotomy: 
The 3-Triangle Method to Correct Axial Alignment and Tibial Slope. The American Journal of Sports Medicine, 33, 378-387. https://doi.org/10.1177/0363546504269034

[27] Song, E.K., Seon, J.K. and Park, S.J. (2007) How to Avoid Unintended Increase of Posterior Slope in Navigation-Assisted Open-Wedge High Tibial Osteotomy. Orthopedics, 30, S127-S131.

[28] Asada, S., et al. (2012) Increase in Posterior Tibial Slope Would Result in Correction Loss in Frontal Plane after Medial Open-Wedge High Tibial Osteotomy. Knee Surgery, Sports Traumatology, Arthroscopy, 20, 571-578. https://doi.org/10.1007/s00167-011-1610-1

[29] Lustig, S., Scholes, C.J., Costa, A.J., Coolican, M.J. and Parker, D.A. (2013) Different Changes in Slope between the Medial and Lateral Tibial Plateau after Open-Wedge High Tibial Osteotomy. Knee Surgery, Sports Traumatology, Arthroscopy, 21, 32-38. https://doi.org/10.1007/s00167-012-2229-6

[30] Dejour, H., et al. (1990) Dysplasia of the Femoral Trochlea. Revue de Chirurgie Orthopedique et Reparatrice de 1 Appareil Moteur, 76, 45-54.

[31] Hoell, S., Suttmoeller, J., Stoll, V., Fuchs, S. and Gosheger, G. (2005) The High Tibial Osteotomy, Open versus Closed Wedge, a Comparison of Methods in $108 \mathrm{~Pa}$ tients. Archives of Orthopaedic and Trauma Surgery, 125, 638-643. https://doi.org/10.1007/s00402-005-0004-6

[32] Bombaci, H., et al. (2005) The Effect of Open Wedge Osteotomy on the Posterior Tibial Slope. Acta Orthopaedica et Traumatologica Turcica, 39, 404-410.

[33] Ozalay, M., et al. (2008) The Correlation of Correction Magnitude and Tibial Slope Changes Following Open Wedge High Tibial Osteotomy. Knee Surgery, Sports Traumatology, Arthroscopy, 16, 948-951. https://doi.org/10.1007/s00167-008-0589-8

[34] Amzallag, J., Pujol, N., Maqdes, A., Beaufils, P., Judet, T. and Catonne, Y. (2013) Patellar Height Modification after High Tibial Osteotomy by Either Medial Opening-Wedge or Lateral Closing-Wedge Osteotomies. Knee Surgery, Sports Traumatology, Arthroscopy, 21, 255-259. https://doi.org/10.1007/s00167-012-2304-Z

[35] Brinkman, J.M., Luites, J.W.H., Wymenga, A.B. and van Heerwaarden, R.J. (2010) Early Full Weight Bearing Is Safe in Open-Wedge High Tibial Osteotomy: RSA Analysis of Postoperative Stability Compared to Delayed Weight Bearing. Acta Orthopaedica, 81, 193-198. https://doi.org/10.3109/17453671003619003

[36] Niemeyer, P., et al. (2010) Open-Wedge Osteotomy Using an Internal Plate Fixator in Patients with Medial-Compartment Gonarthritis and Varus Malalignment: 3-Year Results with Regard to Preoperative Arthroscopic and Radiographic Findings. Arthroscopy. The Journal of Arthroscopic and Related Surgery, 26, 1607-1616. 